\title{
Avaliação da Alexitimia e Percepção Emocional: Comparação entre Autorrelato e Desempenho
}

\author{
Ana Carolina Zuanazzi \\ Programa de Pós-Graduação em Psicologia Clínica da Universidade de São Paulo, \\ São Paulo, SP, Brasil \\ Diana Santos Ricci \\ Universidade Estadual de Londrina, Londrina, PR, Brasil \\ Fabiano Koich Miguel ${ }^{1}$ \\ Departamento de Fundamentos de Psicologia e Psicanálise da Universidade Estadual \\ de Londrina, Londrina, PR, Brasil
}

\begin{abstract}
Resumo
A alexitimia é um quadro em que o indivíduo apresenta prejuízo na capacidade de fantasiar e na capacidade de identificar suas próprias emoções e nos outros. Parte dos resultados conflitantes nessa área pode ser devida à forma de avaliação. A presente pesquisa teve como objetivo comparar dois tipos de instrumentos relacionados à avaliação da alexitimia. Participaram 405 pessoas, que responderam a uma escala de alexitimia (autorrelato) e um teste de percepção de emoções (desempenho). A escala, desenvolvida para esta pesquisa com base na literatura, apresentou uma estrutura de cinco fatores: descrição dos sentimentos, expressão emocional, abstração, percepção emocional e planejamento. Já o teste apresentava vídeos de rostos de pessoas, devendo-se identificar as emoções presentes. Embora as características psicométricas da escala estivessem adequadas, não foram encontradas correlações significativas entre os instrumentos, mesmo no fator de percepção emocional. Os resultados sugerem que o autorrelato é um construto diferente do desempenho. As implicações para avaliação psicológica e alexitimia são discutidas.
\end{abstract}

Palavras-chave: Alexitimia, avaliação psicológica, expressões faciais.

\section{Alexithymia and Emotional Perception Assessment: Comparison between Self-Report and Performance}

\begin{abstract}
Alexithymia is a condition in which the individual has impaired ability to fantasize and impaired ability to identify their own emotions and others'. Part of the discrepant results in this field may be due to the assessment format. This study aimed to compare two types of alexithymia assessment instruments. The participants were 405 people responding to an alexithymia scale (self-report) and a test of emotional perception (performance). The scale was developed for this research based on the literature, and it presented a five factors structure: description of feelings, emotional expression, abstraction, emotional per-
\end{abstract}

Endereço para correspondência: Universidade Estadual de Londrina, Departamento de Psicologia e Psicanálise, Centro de Ciências Biológicas, Caixa Postal 10011, Londrina, PR, Brasil 86057-970. E-mail: anacarolina.zf@ gmail.com,dianna_ricci@hotmail.com e fabiano@avalpsi.com.br

Os autores agradecem a colaboração da turma 60 (2010-2014) de Psicologia da Universidade Estadual de Londrina na criação da escala de alexitimia e coleta de dados. 
ception and planning. The test showed videos of people's faces, and the participant should identify the emotions present. Although the psychometric characteristics of the scale were adequate, no significant correlations were found between the instruments, even with the emotional perception factor. The results suggest that self-report is a different construct from performance. The implications for psychological assessment and alexithymia are discussed.

Keywords: Alexithymia, psychological assessment, facial expressions.

\section{Evaluación de la Alexitimia y de la Percepción Emocional: Comparación entre el Autoinforme y el Desempeño}

\section{Resumen}

La alexitimia es una condición en la que una persona tiene deficiencia en la capacidad de fantasear y en la capacidad de identificar sus propias emociones y en otros. Parte de los resultados contradictorios en esta área puede ser debido a la forma de evaluación. Este estudio tuvo como objetivo comparar dos tipos de instrumentos de evaluación relacionados con la alexitimia. Participaron 405 personas que respondieron a una escala de alexitimia (autoinforme) y una prueba de la percepción de las emociones (desempeño). La escala, desarrollada para esta investigación con base en la literatura, presentó una estructura de cinco factores: descripción de los sentimientos, expresión emocional, abstracción, percepción emocional y planificación. La prueba mostraba videos de rostros de personas, identificándose las emociones presentes. Aunque las características psicométricas de la escala eran adecuadas, no se encontraron correlaciones significativas entre los instrumentos, incluso el factor de la percepción emocional. Los resultados sugieren que el autoinforme es un constructo diferente de desempeño. Se discuten las implicaciones para la evaluación psicológica y la alexitimia.

Palabras clave: Alexitimia, evaluación psicológica, expresiones faciales.

O conceito de alexitimia foi inicialmente elaborado para descrever pacientes com doenças psicossomáticas que apresentavam uma vida emocional sem fantasias e com certa dificuldade em experienciar, demonstrar ou nomear emoções. O termo foi proposto para se referir ao fato da pessoa não ter palavras para descrever as emoções. Com o tempo, o uso do termo alexitimia foi expandido para definir um atributo que não era encontrado apenas em pacientes com doenças psicossomáticas, mas também em pacientes com transtornos psíquicos, como a depressão e o estresse pós-traumático, ou até mesmo em parte da população geral. Sua definição seria então de dificuldade no reconhecimento do estado emocional, reduzida capacidade de fantasiar e interesse voltado a eventos externos (Carneiro \& Yoshida, 2009; Freire, 2010; Veríssimo, 2011).

Embora tenha sido definida há mais de quatro décadas, ainda não encontra um consenso entre os pesquisadores sobre a etiologia da alexitimia. Entende-se que existem diversos fatores envolvidos, que podem ser genéticos, fisiológicos, neuroanatômicos, psicossociais (ou de desenvolvimento) ou neuroquímicos (Freire, 2010; Maciel \& Yoshida, 2006; Sifneos, 1991). Com o desenvolvimento das neurociências, foram desenvolvidas pesquisas com exames de neuroimagem que permitiram compreender melhor as características neurológicas cognitivas e afetivas da alexitimia. Com base nesse modelo, existiriam dois tipos de alexitimia. $\mathrm{Na}$ alexitimia de tipo I, componentes afetivos e cognitivos das experiências vivenciadas estariam comprometidos neurologicamente, por meio de lesões em algumas áreas específicas do cérebro, responsáveis pelo processo de avaliação da experiência emocional, como o hemisfério direito e estruturas pré-frontais. Já na alexitimia de tipo II, apenas os componentes cognitivos das emoções estariam prejudicados. $\mathrm{O}$ indivíduo, nesse caso, vivenciaria experiências emocionais e reações fi- 
siológicas (reposta autonômica), porém não conseguiria interpretar cognitivamente a emoção e as respostas experienciadas (Carneiro \& Yoshida, 2009; Freire, 2010; Wagner \& Lee, 2008).

Para se medir e avaliar alexitimia, alguns métodos são comumente utilizados, como: Beth Israel Hospital Psychosomatic Questionnaire (BIQ), escalas de autorrelato como a escala de alexitimia do MMPI (Minnesota Multiphasic Personality Inventory) e a Schalling-Sifneos Personality Scale (SSPS). Porém, segundo Yoshida (2007), a maioria dos instrumentos não apresenta propriedades psicométricas totalmente aceitáveis. Já a Toronto Alexithymia Scale (TAS), nos estudos da autora, demonstrou resultados compatíveis com o construto de alexitimia observado por clínicos. A autora e colegas realizaram pesquisas no Brasil com a TAS, verificando a adequação psicométrica da escala (Yoshida, 2000, 2007). Foram identificadas quatro dimensões na TAS: identificação e distinção dos sentimentos; uso de fantasias; foco em eventos externos em vez de internos; e comunicação dos sentimentos.

Já Bermond et al. (2007), analisando a escala Bermond-Vorst Alexithymia Questionnaire (BVAQ), chegaram a cinco fatores: capacidade de fantasiar, sonhar acordado e fazer projetos; excitabilidade emocional; percepção emocional; descrição externa dos sentimentos; e comunicação dos sentimentos. Os dois primeiros representariam uma dimensão afetiva e os três últimos, cognitiva. A BVAQ, assim como os instrumentos apresentados anteriormente, possui o formato de autorrelato, em que o próprio indivíduo atribui uma pontuação para as diferentes escalas.

Em pesquisas, os instrumentos de avaliação têm sido utilizados para se compreender a relação da alexitimia com diversos transtornos e doenças. Nesse contexto, a alexitimia se configura como um possível fator de risco em algumas condições (por exemplo, o uso abusivo de substâncias psicoativas) ou como um traço presente em transtornos psiquiátricos ou em pacientes com doenças psicossomáticas. A seguir serão expostos alguns dos estudos que buscaram fazer essa relação.
Em um estudo americano, Krystal, Giller e Cicchetti (1986) aplicaram quatro instrumentos para avaliar o grau de alexitimia em 45 pacientes divididos em quatro grupos. Dois grupos foram compostos por veteranos que lutaram na guerra do Vietnam diagnosticados com transtorno de estresse pós-traumático, sendo que um grupo foi composto por pacientes internados, outro por pacientes que recebiam tratamento ambulatorial, o terceiro grupo por pacientes com alguma doença psicossomática e o último grupo por pacientes com transtornos afetivos variados. Apesar dos resultados apontarem para um grau mais elevado de alexitimia em veteranos do Vietnam internados e pacientes com alguma doença psicossomática, estudos de correlação entre os instrumentos utilizados para avaliar alexitimia apresentaram correlações baixas e até mesmo negativas entre si.

Num estudo italiano, Porcelli et al. (2004) avaliaram e compararam, entre outras variáveis, o grau de alexitimia em um grupo de pacientes psiquiátricos e um grupo de pacientes diagnosticados com alguma desordem funcional gastrointestinal. Por meio da TAS-20, foi constatado que o grupo de indivíduos com alguma desordem funcional gastrointestinal apresentou escores significativamente mais elevados de alexitimia quando comparado com o grupo de pacientes psiquiátricos. Cerca de $75 \%$ do grupo com desordens gastrointestinais obteve escore alto em alexitimia e apenas $21 \%$ do grupo de pacientes psiquiátricos obteve escore elevado em alexitimia. O estudo aponta, portanto, para uma possível relação entre alexitimia e desordens funcionais gastrointestinais.

No Brasil, um estudo realizado por Maciel e Yoshida (2006) avaliou e comparou, entre outras variáveis, o grau de alexitimia em homens diagnosticados como dependentes de álcool e homens não dependentes de álcool, de acordo com a Classificação Internacional de Doenças (CID10). Foram aplicados três instrumentos, dentre eles a TAS-26. Em relação à alexitimia, dois dos quatro fatores avaliados pela TAS foram considerados significativamente mais elevados em indivíduos dependentes de álcool quando comparados a indivíduos não dependentes, que dizem respeito a dificuldades em descrever sentimentos 
e diminuição do uso de fantasias. Porém, nos outros dois fatores avaliados pela escala não foram encontradas diferenças significativas entre os grupos. Nesse estudo, algumas considerações sobre os itens utilizados na TAS-26 foram feitas, além da proposta de exclusão de um dos fatores avaliados pela escala.

Em outro estudo brasileiro, Oliveira e Yoshida (2009) realizaram uma bateria de testes em pacientes de uma lista de espera para cirurgia bariátrica e em pacientes que haviam se submetido à cirurgia há pelo menos seis meses. Dentre os instrumentos aplicados, usou-se a TAS-26 para avaliar o grau de alexitimia nos dois grupos, e foi constatada diferença significativa entre grupos apenas no fator relacionado a identificação dos sentimentos. Além disso, considerando-se a pontuação geral média de cada grupo, não foi constatado a presença de alexitimia segundo o critério adotado pelas autoras. Apenas uma pequena porcentagem de participantes de ambos os grupos (cerca de $28 \%$ no grupo em espera e $9 \%$ no grupo com cirurgia) apresentaram pontuação individual que se enquadraria na classificação de alexitimia. As autoras especularam que o fato de haver uma incidência maior de alexitímicos no primeiro grupo poderia estar relacionado ao impacto emocional que a obesidade pode exercer sobre as pessoas, porém não há estudos que confirmem a relação entre alexitimia e obesidade.

Em outro estudo italiano, Nicolo et al. (2011) buscaram correlacionar sintomas de transtornos de personalidade com alexitimia. $\mathrm{O}$ estudo reuniu 388 participantes com variados diagnósticos de transtornos de personalidade. Os participantes responderam a TAS-20 e foram classificados em três grupos: não-alexitímicos, intermediários e alexitímicos. Foi constatado que em alguns transtornos pode-se relacionar os respectivos sintomas do transtorno com a presença de alexitimia (por exemplo, nos casos de depressão), porém, em outros casos (como nos transtornos obsessivos compulsivos), essa relação não foi encontrada.

Em um estudo português, Torres et al. (2011) pesquisaram a incidência de alexitimia em pacientes com anorexia nervosa. $\mathrm{O}$ instrumento TAS-20 foi utilizado e constatou-se que, dos 80 participantes com anorexia nervosa, cerca de $62 \%$ apresentavam alexitimia. Esse número, segundo os autores, é significativamente superior quando comparado a pessoas que não tinham anorexia nervosa. Outro dado encontrado é que o nível de escolaridade também estaria associado a presença alexitimia, no sentido de, quanto menor o nível de escolaridade, mais frequentemente se constatou alexitimia em pacientes. Os autores concluem que a alexitimia é uma característica saliente naquele transtorno, independentemente da sua severidade.

Os estudos apresentados, apesar de terem sido desenvolvidos com diferentes populações, têm em comum a tentativa de relacionar a alexitimia a diferentes transtornos. Nota-se, porém, que alguns estudos não apresentam dados que possibilitem afirmar concretamente essa relação, ou pelo menos não de maneira inequívoca. Alguns fatores podem contribuir para essa situação, como a própria dificuldade em se avaliar a alexitimia. Além disso, os instrumentos utilizados para avaliar o mesmo construto, em alguns casos, mostraram-se estatisticamente contraditórios.

Partindo dessas informações, a presente pesquisa levantou a hipótese de que o tipo de instrumento utilizado para avaliação pode influenciar no resultado obtido. Assim, teve-se como objetivo estudar a relação entre dois tipos de instrumentos, um por autorrelato e outro por desempenho, destinados à avaliação de aspectos da alexitimia. Mais especificamente, na pesquisa foi desenvolvida uma escala de autorrelato e comparada com um teste de percepção emocional em vídeos de pessoas.

\section{Método}

\section{Participantes}

Participaram da pesquisa 405 pessoas, sendo $220(54,3 \%)$ do gênero feminino, 152 $(37,5 \%)$ masculino e $33(8,1 \%)$ que não informaram. A idade média foi 26,2 $(D P=8,9)$. Em relação àqueles que informaram a escolaridade, haviam participantes cursando do $7^{\circ}$ ano do ensino fundamental até a pós-graduação, sendo que $22,2 \%$ estavam cursando até o $3^{\circ}$ ano do ensino 
médio, $59,6 \%$ cursavam ou tinham curso ensino superior e $18,2 \%$ estavam realizando ou tinham pós-graduação. Do total, 206 participantes relataram a formação de nível superior, sendo de 50 cursos diferentes. Dentre eles, $22,8 \%$ relataram Psicologia, $14,1 \%$ Direito, 4,4\% Informática, $3,4 \%$ Veterinária, e cada curso restante teve participação de menos de 3\%. Das 273 pessoas que informaram o estado onde residiam, 74,7\% eram do Paraná, 16,8\% de São Paulo, ficando os estados Espírito Santo, Goiás, Minas Gerais, Pará, Paraíba, Rio de Janeiro, Rondônia e Santa Catarina com menos de $2 \%$ cada.

\section{Instrumentos}

A Escala de Psicologia de Alexitimia (EPA) foi composta por 38 itens no formato de autorrelato, com pontuação em escala Likert. O participante era instruído a pontuar cada item de acordo com a seguinte escala: 1 - Nunca (a situação nunca ou quase nunca aconteceu comigo), 2 - Poucas vezes (a situação já aconteceu comigo, mas foram poucas vezes), 3 - Muitas vezes (a situação já aconteceu comigo, e foram várias vezes), 4 - Sempre (a situação sempre ou quase sempre aconteceu comigo). Alguns itens foram escritos em sentido invertido, ou seja, expressando uma facilidade em vez de dificuldade. A pontuação de tais itens foi invertida para estudo da escala.

O Teste Informatizado de Percepções de Emoções Primárias (PEP) se propõe a avaliar a capacidade de reconhecer adequadamente as expressões emocionais em outras pessoas. Para tanto, é composto por 38 itens que apresentam, na forma de vídeo, pessoas expressando uma ou mais emoções, sendo que os três primeiros são exemplos para que o avaliando se familiarize com a tarefa proposta. Cada vídeo possui de 3 a 8 segundos de duração. A tarefa é responder qual ou quais emoções estão sendo apresentadas em cada vídeo, dentro de um rol de oito estados emocionais (alegria, amor, medo, surpresa, tristeza, nojo, raiva e curiosidade). O PEP vem sendo estudado em relação a critérios e construtos semelhantes ou divergentes, e apresenta evidências de validade favoráveis (Miguel, Finoto, \& Miras, 2013; Miguel, Ogaki, Inaba, \& Ribeiro, 2013; Miguel \& Primi, 2014).

\section{Procedimentos}

Construção da Escala. A EPA foi desenvolvida na disciplina Estágio em Formação de Bacharelado em Psicologia, que acontece no quarto ano da graduação, ministrada pelo terceiro autor desta pesquisa. Como atividade da disciplina, os estudantes fizeram a leitura de publicações sobre alexitimia (que constam na introdução teórica) e foram organizados em grupos de maneira que cada grupo contribuísse com itens para a composição da escala. Inicialmente foram desenvolvidos 92 itens. Durante encontros da disciplina, os estudantes, supervisionados pelo professor com formação em construção de instrumentos de avaliação, agruparam e analisaram os itens quanto ao seu conteúdo e relação com a descrição do construto. Foi discutida a presença de itens com redação muito semelhante, como, por exemplo, "Acho difícil explicar meus sentimentos para os outros", "Eu encontro dificuldades para descrever como estou me sentindo", "Tenho dificuldades para comunicar verbalmente o que sinto para as demais pessoas". Assim, itens muito próximos de outros foram excluídos. Ao final, 38 itens foram selecionados para compor a EPA.

Coleta de Dados. A escala foi formatada para aplicação online e armazenada em um domínio de pesquisas em avaliação psicológica, onde também se encontrava o PEP. A pesquisa foi cadastrada no Sistema Nacional de Informação sobre Ética em Pesquisa (SISNEP) e aprovada pelo Comitê de Ética e Pesquisa da Universidade Estadual de Londrina (protocolo 0159.0.268.000-10). Os participantes foram contatados tanto pessoalmente quanto por mídia eletrônica, recebendo o link da pesquisa e um código individual de acesso. Os resultados dos participantes foram armazenados em banco de dados para se realizar as análises psicométricas da EPA e estudo da relação com PEP.

Análise de Dados. Quanto ao funcionamento da escala, foram realizadas análises fatoriais exploratórias, a fim de compreender a dimensionalidade. Os fatores foram individualmente estudados quanto às dificuldades dos itens, ajuste $\mathrm{e}$ precisão segundo a Teoria de Resposta ao Item, mais especificamente o modelo de Rasch, por meio do software Winsteps 3.69.1.7. 
A dificuldade do item é uma medida numérica diretamente relacionada ao construto avaliado pelo teste, e ambos são apresentados em uma mesma escala. Por exemplo, um indivíduo com medida 1,00 tenderia a endossar (atribuir pontuações maiores em) um item com dificuldade $-1,00$, e tenderia a rejeitar (atribuir pontuações menores em) um item com dificuldade 3,00. Já a precisão é medida tanto por meio de um índice geral do teste quanto individualmente nos itens. No caso, foi verificada a correlação item-total, que indica quanto o item contribui para a medida final daquela escala. Itens com correlações maiores estão mais relacionados ao construto medido. Por fim, as medidas de ajuste verificam o quanto o modelo de Rasch explica os dados reais observados. Segundo o manual do software (Linacre, 2009), itens com índices de desajuste acima de 1,50 devem ser utilizados com cautela, enquanto desajuste acima de 2,00 são prejudiciais para a avaliação.

Tanto os resultados da EPA quanto do PEP foram calculados segundo modelo de Rasch. Para se comparar os instrumentos, utilizou-se correlação de Spearman. Optou-se por este método porque alguns fatores da EPA, provavelmente devido ao baixo número de itens, não alcançaram distribuição próxima da normal.

\section{Resultados}

Uma primeira análise de precisão da EPA foi realizada, com o propósito de identificar itens que apresentaram baixa correlação item-total. $\mathrm{O}$ alfa de Cronbach da escala geral foi de 0,85 e os seguintes itens tiveram correlações inferiores a 0,20: $13(r=0,17), 16(r=0,06), 25(r=0,14)$, $32(r=0,09), 36(r=0,12)$. Considerando que os índices indicavam baixa contribuição na avaliação do construto, esses cinco itens foram então removidos das subsequentes análises, ficando a escala com um total de 33 itens.

Prosseguiu-se análise fatorial exploratória, visando identificar as dimensões presentes no instrumento. O índice Kaiser-Meyer-Olkin foi de 0,85 e o teste de esfericidade de Bartlett foi significativo no nível 0,000 , indicando que os dados estavam adequados para esse tipo de análise. O gráfico de sedimentação (scree plot) está apresentado na Figura 1.

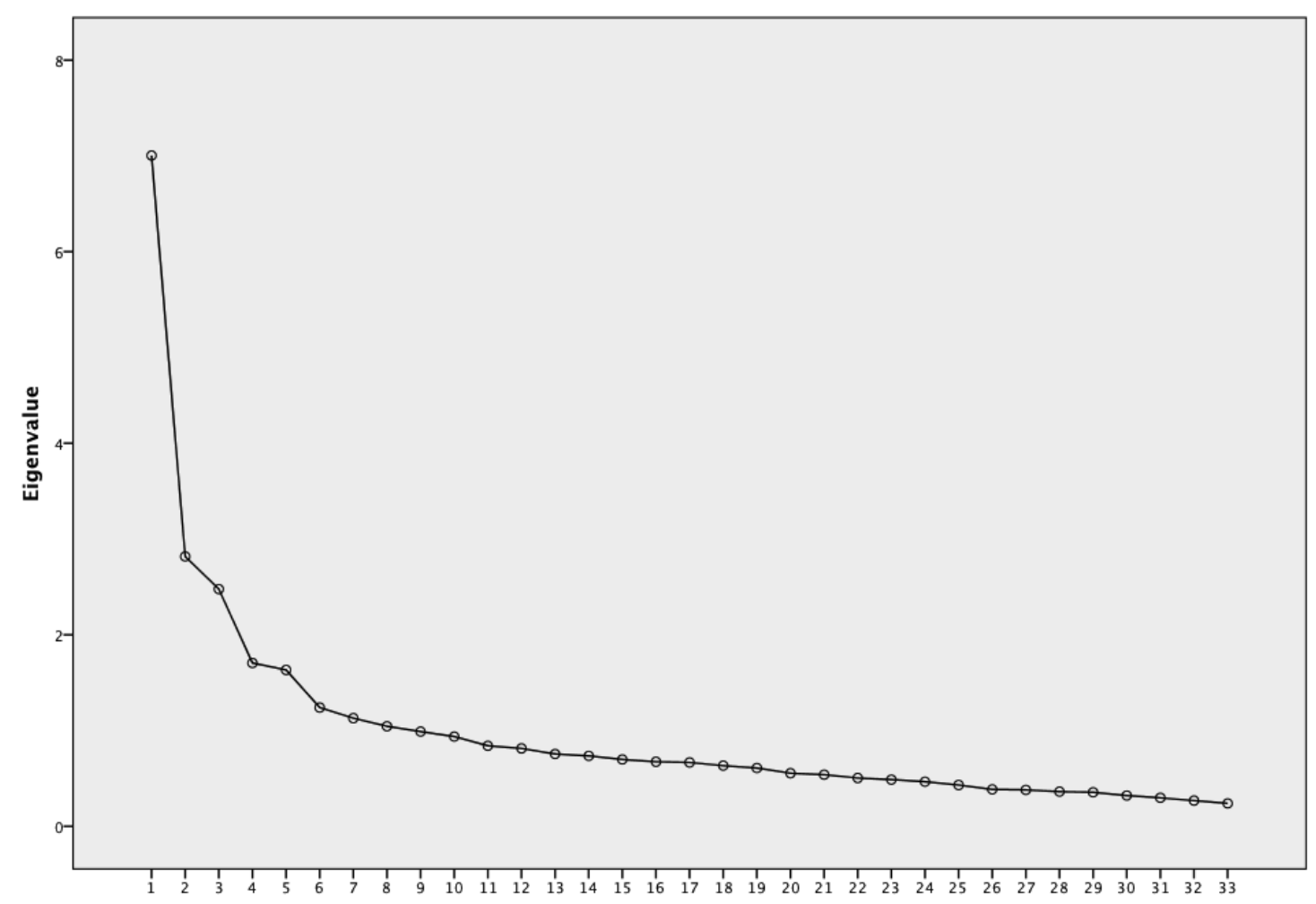

Figura 1. Gráfico de sedimentação (scree plot) dos 33 itens da Escala de Psicologia de Alexitimia (EPA). 
Embora exista uma quantidade de fatores com eigenvalue acima de 1 , a inspeção visual mostrou estabilização da diferença de eigenvalues a partir de seis fatores. Com base nisso, foi realizada extração de seis fatores utilizando-se rotação oblimin. Foi possível perceber que apenas três itens compuseram o sexto fator, e todos também possuíam cargas em outros fatores. Por esse motivo, optou-se por manter a estrutura de cinco fatores, uma vez que se assemelhavam à estrutura encontrada na escala BVAQ por Bermond et al. (2007).

O primeiro fator apresentou eigenvalue 7,01 com variância de 21,23; o segundo fator apresentou eigenvalue 2,82 com variância de 8,54; o terceiro fator apresentou eigenvalue 2,48 com variância de 7,51; o quarto fator apresentou eigenvalue 1,71 com variância de 5,17 ; o quinto fator apresentou eigenvalue 1,63 com variância de 4,95. A variância acumulada foi de 47,39. Embora ainda não exista um valor considerado adequado para a variância total explicada, a EPA aproxima-se da média encontrada na literatura (para uma revisão do tema, ver Damásio, 2012).

Os itens foram distribuídos conforme a Tabela 1 e a Tabela 2. Dessa forma, foi possível perceber coerência no conteúdo dos itens de cada fator, atribuindo-se os seguintes títulos para cada um: 1 - Descrição de emoções, 2 - Expressão de emoções, 3 - Abstração, 4 - Percepção de emoções, 5 - Planejamento.

Tabela 1

Características Psicométricas dos Itens dos Fatores 1 e 2, segundo Modelo de Rasch

\begin{tabular}{|c|c|c|c|c|}
\hline Item & $\begin{array}{l}\text { Dificul- } \\
\text { dade }\end{array}$ & Infit & Outfit & $\begin{array}{l}\text { Corr. } \\
\text { item total }\end{array}$ \\
\hline \multicolumn{5}{|l|}{ Fator 1 - Descrição } \\
\hline 01 - Acho difícil explicar meus sentimentos para os outros & $-0,80$ & 1,05 & 1,09 & 0,62 \\
\hline 03 - Fico em dúvida quando tenho que escolher algo & $-1,54$ & 1,16 & 1,17 & 0,57 \\
\hline 04 - Quando fico com raiva, não sei descrever o motivo & 0,82 & 0,97 & 0,94 & 0,65 \\
\hline $\begin{array}{l}14 \text { - Tenho dificuldade em perceber o que estou sentindo em } \\
\text { determinadas situações }\end{array}$ & 0,18 & 0,80 & 0,81 & 0,72 \\
\hline 19 - Quando me emociono, sempre sei descrever o que estou sentindo (I) & 0,25 & 1,05 & 1,06 & 0,62 \\
\hline 26 - Não consigo entender por que tenho certos sentimentos & $-0,31$ & 1,01 & 1,02 & 0,64 \\
\hline 27 - Quando me perguntam como estou me sentindo, eu sei explicar (I) & 0,23 & 0,84 & 0,84 & 0,71 \\
\hline 28 - Tenho sensações corporais que não sei nomear & 0,75 & 1,11 & 1,12 & 0,58 \\
\hline 30 - Frequentemente não sei identificar o que quero fazer & 0,52 & 1,10 & 1,10 & 0,60 \\
\hline 35 - Eu encontro dificuldades para colocar em palavras o que estou sentindo & $-0,09$ & 0,84 & 0,84 & 0,71 \\
\hline \multicolumn{5}{|l|}{ Fator 2 - Expressão } \\
\hline 02 - Eu sou uma pessoa que dá poucas risadas & 0,77 & 1,40 & 1,60 & 0,42 \\
\hline $\begin{array}{l}07 \text { - Quando tento expressar meus sentimentos, as pessoas dizem que } \\
\text { não me entendem }\end{array}$ & $-0,11$ & 1,01 & 1,00 & 0,59 \\
\hline 08 - Pessoas que são muito emotivas me incomodam & $-0,33$ & 1,38 & 1,41 & 0,47 \\
\hline 15 - Eu expresso poucas emoções & $-0,38$ & 0,84 & 0,84 & 0,71 \\
\hline 18 - Não sei quando expressar minhas emoções & $-0,22$ & 0,92 & 0,92 & 0,65 \\
\hline 21 - As pessoas dizem que sou muito fechado & $-0,31$ & 0,78 & 0,79 & 0,74 \\
\hline 33 - As pessoas dizem que nunca expresso minhas emoções e sentimentos & 0,20 & 0,71 & 0,72 & 0,75 \\
\hline $\begin{array}{l}38 \text { - As pessoas dizem que não sabem o que estou sentindo e, por isso, } \\
\text { não me compreendem }\end{array}$ & 0,37 & 0,94 & 0,93 & 0,64 \\
\hline
\end{tabular}

Nota. (I) Item de pontuação invertida. 
Com a estrutura fatorial definida, os itens foram analisados por meio da Teoria de Resposta ao Item, de acordo o modelo de Rasch. Os resultados dos fatores 1 - Descrição e 2 - Expressão são apresentados na Tabela 1. O código (I) indica que se trata de item de pontuação invertida. A precisão do fator 1 foi 0,86 e do fator 2 foi 0,80 , considerados índices bons. Também se pode perceber que todos os indicadores de desajuste dos itens permaneceram abaixo do recomendado. $\mathrm{O}$ item 02 do segundo fator apresentou outfit de 1,60. Assim mesmo, optou-se por manter o item no teste, pois seu infit foi dentro do adequado e sua dificuldade foi relativamente mais alta, contribuindo para medição de níveis mais severos do construto.

Como exemplo da distribuição dos itens, o que apresentou menor dificuldade no primei- ro fator foi "Fico em dúvida quando tenho que escolher algo", mostrando que essa situação costuma ser endossada com maior frequência pelas pessoas. Já o item com maior dificuldade foi "Quando fico com raiva, não sei descrever o motivo", sendo menos frequente de ser endossado. A quantidade do construto avaliado está diretamente relacionada com as dificuldades dos itens. Assim, atribuir nota 4 para o item mais difícil representa uma maior quantidade do construto latente do que atribuir nota 4 para o item mais fácil.

Os resultados dos fatores 3 - Abstração, 4 Percepção e 5 - Planejamento são apresentados na Tabela 2. A precisão do fator 3 foi 0,75 , do fator 4 foi 0,64 e do fator 5 foi 0,59 . Os dois últimos fatores tiveram índices de precisão não tão adequados. Por outro lado, são fatores com pre-

Tabela 2

Características Psicométricas dos Itens dos Fatores 3, 4 e 5, segundo Modelo de Rasch

\begin{tabular}{lcccc}
\hline \multicolumn{1}{c}{ Item } & $\begin{array}{c}\text { Dificul- } \\
\text { dade }\end{array}$ & $\begin{array}{c}\text { Infit } \\
\text { Outfit }\end{array}$ & $\begin{array}{c}\text { Corr. } \\
\text { item total }\end{array}$ \\
\hline Fator 3 - Abstração & & & & \\
06 - Prefiro atividades manuais do que ficar imaginando coisas & $-1,01$ & 1,15 & 1,18 & 0,50 \\
10 - Acho que ficar filosofando sobre o motivo das coisas é perda de tempo & 0,39 & 1,06 & 1,03 & 0,53 \\
11 - Ditados populares e metáforas são difíceis de entender & 0,88 & 0,91 & 0,87 & 0,57 \\
17 - Não considero importante entender como as coisas funcionam & 0,70 & 1,11 & 1,26 & 0,45 \\
20 - Gosto de metáforas pois elas facilitam entender as coisas (I) & $-0,69$ & 1,09 & 1,10 & 0,55 \\
29 - Tenho dificuldade em entender piadas & 0,41 & 1,07 & 1,11 & 0,48 \\
31 - Se as pessoas não forem objetivas, acho difícil entender o que elas querem & $-0,79$ & 1,05 & 1,09 & 0,52 \\
$34-$ Tenho dificuldade para entender coisas abstratas e filosóficas & 0,03 & 0,74 & 0,73 & 0,71 \\
37 - Não gosto de atividades que envolvam interpretações ou reflexões & 0,08 & 0,82 & 0,80 & 0,66
\end{tabular}

Fator 4 - Percepção

05 - Consigo identificar facilmente quando as pessoas têm "segundas intenções” (I) $\quad-0,42 \quad 1,04 \quad 1,05 \quad 0,70$

22 - Consigo identificar facilmente o que os outros estão sentindo (I)

$\begin{array}{llll}-0,37 & 0,90 & 0,90 & 0,72\end{array}$

24 - Tenho facilidade para imaginar situações alegres ou tristes (I)

$0,79 \quad 1,03 \quad 1,06 \quad 0,68$

Fator 5 - Planejamento

09 - Não gosto de ficar planejando meu futuro

$0,41 \quad 0,86 \quad 0,84 \quad 0,72$

12 - Gosto de trabalhos em que eu tenha que tomar as decisões (I)

$\begin{array}{llll}-0,89 & 1,37 & 1,36 & 0,61\end{array}$

23 - Tenho muitos planos para o futuro (I)

$0,48 \quad 0,74 \quad 0,73 \quad 0,76$

Nota. (I) Item de pontuação invertida. 
sença de poucos itens, e sabe-se que a precisão de uma escala é diretamente relacionada à sua quantidade de itens. Nesse sentido, a precisão do modelo de Rasch ainda mostrou-se superior à precisão por meio do alfa de Cronbach para esses dois fatores, que foram 0,49 para fator $4 \mathrm{e}$ 0,50 para fator 5 . Também não houve itens com índices de desajuste acima de 2,00.

Com as características psicométricas dos itens, foram calculadas as pontuações dos participantes nos cinco fatores da EPA e também no PEP. Os resultados estão apresentados na parte superior da Tabela 3. Pode-se perceber uma ampla distribuição de pontuações em todas as escalas.
Em seguida foram realizadas correlações de Spearman com os fatores da EPA entre si e o PEP. Foram 372 (91,9\%) participantes que responderam ambos os instrumentos. Os resultados são apresentados na parte inferior da Tabela 3. Houve correlações significativas de todos os fatores da EPA entre si, algumas em nível moderado a alto, outras em nível leve. Esses resultados indicam que, apesar do construto avaliado pela escala não ser unidimensional, compõe-se de traços que são interrelacionados, o que era esperado. Já no que diz respeito à relação da EPA com PEP, as cinco correlações foram extremamente baixas - próximas do nulo - e não significativas. Esses resultados indicam a não existência de relação entre a capacidade de perceber emoções no PEP e cada uma das escalas da EPA.

Tabela 3

Estatística Descritiva dos Participantes e Correlações de Spearman entre Fatores da EPA e PEP

\begin{tabular}{ccccccc}
\hline & EPA1 & EPA2 & EPA3 & EPA4 & EPA5 & PEP \\
Média & $-0,48$ & $-0,93$ & $-1,09$ & $-1,40$ & $-1,23$ & 0,47 \\
Desvio-padrão & 1,48 & 1,37 & 1,17 & 2,03 & 1,70 & 0,79 \\
Mínimo & $-5,53$ & $-5,66$ & $-5,94$ & $-6,59$ & $-4,88$ & $-2,76$ \\
Máximo & 3,32 & 2,72 & 1,83 & 4,49 & 4,47 & 7,06 \\
EPA1 - Descrição & & & & & 0,05 \\
EPA2 - Expressão & $0,50^{*}$ & & & & & $-0,07$ \\
EPA3 - Abstração & $0,31^{*}$ & $0,26^{*}$ & & & & $-0,08$ \\
EPA4 - Percepção & $0,26^{*}$ & $0,16^{*}$ & $0,33^{*}$ & & & \\
EPA5 - Planejamento & $0,26^{*}$ & $0,29^{*}$ & $0,18^{*}$ & $0,23 *$ & & \\
\hline
\end{tabular}

Notas. EPA=Escala de Psicologia de Alexitimia; PEP=Teste Informatizado de Percepções de Emoções Primárias; $N=405$ para fatores da EPA; $N=372$ para EPA e PEP; *Correlação significativa no nível $p<0,01$.

Além disso, foi realizada correlação de Spearman entre o PEP e o item 22 da EPA, cuja redação especificamente fala sobre a capacidade de perceber emoções ("Consigo identificar facilmente o que os outros estão sentindo"). O resultado foi rho $=-0,05(p=0,312)$, indicando ausência de relação entre as duas pontuações. Esses resultados reproduzem aqueles encontrados ao se relacionar uma versão preliminar do PEP com a TAS-26 (Miguel, 2010).

\section{Discussão}

A presente pesquisa teve como objetivo a criação de uma escala de autorrelato para avaliação da alexitimia (EPA) e a comparação com um instrumento de avaliação por desempenho da capacidade de perceber emoções em rostos de pessoas (PEP). Parte do procedimento incluiu a criação da escala EPA, que apresentou uma estrutura não unidimensional, o que era 
esperado, uma vez que a descrição de alexitimia é composta por várias características. Tais características mostraram-se representadas nos cinco fatores da escala: dificuldade em descrever os próprios sentimentos (fator 1), dificuldade na expressão emocional (fator 2), dificuldade na compreensão abstrata (fator 3 ), dificuldade em descrever as expressões emocionais dos outros (fator 4) e dificuldade em planejar tarefas (fator 5).

Os critérios para retenção de cinco fatores foram baseados na remoção de fatores com poucos itens e carga duplicada, e também na coerência com a estrutura fatorial da escala $B V A Q$, previamente estudada por Bermond et al. (2007). Por outro lado, ainda não existe consenso na literatura quanto à quantidade de fatores no construto alexitimia, por isso a pertinência de cinco fatores ou qualquer outro número ainda pode ser debatida. Entende-se que outros procedimentos poderiam ser adotados com o propósito de se verificar a adequação da estrutura fatorial. Por exemplo, estudos futuros poderiam utilizar análise paralela a fim de estabelecer uma quantidade mais confiável de fatores ou mesmo análise fatorial confirmatória em outras amostras.

Para cada um dos fatores, foram calculadas as propriedades psicométricas segundo o modelo de Rasch. Foi possível perceber níveis distintos de dificuldades dos itens dentro de cada um dos fatores. Esses resultados ilustram a necessidade de se relativizar o peso de cada item dentro de um instrumento de avaliação psicológica. Por exemplo, um indivíduo que concorda totalmente com um item como "Quando fico com raiva, não sei descrever o motivo" (dificuldade 0,82) provavelmente apresenta maior quantidade de alexitimia do que um indivíduo que assinala nota máxima em "Se as pessoas não forem objetivas, acho difícil entender o que elas querem" (dificuldade -0,79). Um dos benefícios dos modelos de Teoria de Resposta ao Item, como o Rasch, é justamente a produção de uma pontuação individual diretamente relacionada às dificuldades dos itens do teste, contrapondo-se com o modelo tradicional de medição, em que cada item contribui com o mesmo peso para a nota final.
Os índices de precisão dos fatores 4 e 5 foram menores do que os outros, ficando abaixo do recomendável, embora ainda próximos do aceitável. Considerou-se que esse resultado se deveu ao fato de cada fator possuir apenas três itens. Não obstante, esses itens apresentaram elevadas correlações item-total e adequados índices de ajuste, justificando sua presença na escala. Em pesquisas futuras, sugere-se a inclusão de novos itens nesses fatores, a fim de aumentar a precisão da medição.

Além disso, a pesquisa investigou a relação entre a EPA e o PEP. Embora os construtos que ambos medem não sejam idênticos, uma das características da alexitimia é o prejuízo na capacidade de perceber emoções nos outros, foco do PEP. As correlações mostraram-se nulas, não sendo possível encontrar uma relação entre os instrumentos. Mesmo o fator 4, com itens de percepção emocional, e individualmente o item 22 ("Consigo identificar facilmente o que os outros estão sentindo") apresentaram correlações nulas com o PEP. Outros pesquisadores (Mihura, 2012; Paulhus, Lysy, \& Yik, 1998; Woyciekoski \& Hutz, 2010) já encontraram que, em avaliação psicológica, instrumentos que medem o mesmo construto por autorrelato e por desempenho nem sempre apresentam correlações elevadas, sendo frequentemente baixas e até mesmo nulas, especialmente quando se trata de capacidades como a percepção de emoções. O mesmo resultado foi encontrado nesta pesquisa, o que leva a um importante questionamento em relação à utilização de instrumentos de autorrelato, que é sua adequação ao construto medido.

No caso da percepção de emoções, trata-se de uma capacidade, e nem sempre o indivíduo pode ter clareza de quanto possui dela. Perguntar a uma pessoa quão bem ela consegue identificar as emoções, tanto em si quanto nos outros, pode ser semelhante a perguntar se uma pessoa tem bom raciocínio espacial ou tem boa memória. Diversos fatores podem influenciar na sua resposta. Um deles pode ser a própria alexitimia, ou seja, a falta de capacidade de compreender os estados emocionais. Nessas situações, seria possível confiar que um alexitímico 
sempre reconhece que tem essa dificuldade? Ou, justamente pelo fato de não compreender direito, pode acreditar que sua capacidade é mediana ou até mesmo alta? Mesmo em pessoas não alexitímicas podem existir outros fatores influenciando como, por exemplo, a autoestima e a autoeficácia. De fato, os resultados demonstraram que mesmo pessoas com elevada capacidade de perceber emoções no PEP podem tanto afirmar que têm uma percepção muito boa quanto que têm uma percepção muito ruim. Nesta pesquisa, esses outros fatores influenciadores não foram estudados ou controlados, por isso sugere-se que tais informações sejam levadas em conta em futuras pesquisas com alexitimia. Portanto, considerando-se as informações apresentadas, sugere-se cautela na utilização do autorrelato para avaliar a percepção de emoções, especialmente na alexitimia.

Não obstante, nem todas as características da alexitimia necessariamente poderiam ser enviesadas pela forma de avaliação. Questões como "As pessoas dizem que sou muito fechado" (relativo a expressividade emocional) ou "Não gosto de atividades que envolvam interpretações ou reflexões" (abstração) provavelmente são mais confiáveis no autorrelato, pois dizem respeito a opiniões externas objetivas ou gosto pessoal, não dependendo do peso da prova de uma capacidade. Nesse sentido, provavelmente uma avaliação mais completa e adequada da alexitimia fosse aquela que lançasse mão tanto de instrumentos de autorrelato quanto de desempenho.

Sugere-se também que mesmo o autorrelato de capacidades pode se configurar como informação útil ao psicólogo. Por exemplo, pela comparação dos resultados de testes de desempenho e de autorrelato, seria possível uma avaliação da percepção que o indivíduo tem das suas próprias capacidades. Dessa forma, a EPA poderia contribuir com informação sobre a opinião individual da capacidade de identificar de emoções. Uma sugestão de novo nome seria Escala de Percepção de Alexitimia, mantendo a mesma sigla e reafirmando o verdadeiro construto avaliado nesse tipo de instrumento.

\section{Referências}

Bermond, B., Clayton, K., Liberova, A., Luminet, O., Maruszewski, T., Bitti, P. E. R., ...Wicherts, J. (2007). A cognitive and an affective dimension of alexithymia in six languages and seven populations. Cognition \& Emotion, 21(5), 1125 1136. doi:10.1080/02699930601056989

Carneiro, B. V., \& Yoshida, E. M. P. (2009). Alexitimia: Uma revisão do conceito. Psicologia: Teoria e Pesquisa, 25(1), 103-108. doi:10.1590/ S0102-37722009000100012

Damásio, B. F. (2012). Uso da análise fatorial exploratória em Psicologia. Avaliação Psicológica, 11(2), 213-228. Recuperado em http:// pepsic.bvsalud.org/scielo.php?script $=$ sci arttext\&pid=S1677-04712012000200007\&lng= pt\&nrm=iso\&tlng=pt

Freire, L. (2010). Alexitimia: Dificuldade de expressão ou ausência de sentimento? Uma análise teórica. Psicologia: Teoria e Pesquisa, 26(1), 15-24. doi:10.1590/S0102-37722010000100003

Krystal, J. H., Giller, E. L., \& Cicchetti, D. V. (1986). Assessment of alexithymia in posttraumatic stress disorder and somatic illness: Introduction of a reliable measure. Psychosomatic Medicine, 48(1), 84-94. Recuperado em http:// journals.lww.com/psychosomaticmedicine/ Abstract/1986/01000/Assessment_of_alexithymia_in_posttraumatic_stress.7.aspx

Linacre, J. M. (2009). A user's guide to WinstepsMinistep: Rasch-model computer programs. Chicago, IL: winsteps.com.

Maciel, M. J. N., \& Yoshida, E. M. P. (2006). Avaliação de alexitimia, neuroticismo e depressão em dependentes de álcool. Avaliação Psicológica, 5(1), 43-54. Recuperado em http:// pepsic.bvsalud.org/scielo.php?pid=S1677$-04712006000100006 \&$ script=sci_arttext

Miguel, F. K. (2010). Criação e validação de um teste informatizado para avaliar a capacidade de perceber emoções primárias (Tese de doutorado, Programa de Pós-Graduação Stricto Sensu em Psicologia, Universidade São Francisco). Recuperado em http://www.usf.edu.br/itatiba/ mestrado/psicologia/uploadAddress/Fabiano_ koich_Miguel[14166].pdf

Miguel, F. K., Finoto, B. A. S., \& Miras, B. D. (2013). Percepção emocional e traços de personalidade: Estudo de validade divergente. Encontro: Revista de Psicologia, 16(24), 107-120. 
Miguel, F. K., Ogaki, H. A., Inaba, C. M., \& Ribeiro, D. O. (2013). Percepção emocional e inteligência: Contribuições para o modelo $\mathrm{CHC}$. Revista Sul-Americana de Psicologia, 1(1), 3647. Recuperado em http://www.revista.unisal. br/am/index.php/psico/article/view/3

Miguel, F. K., \& Primi, R. (2014). Estudo psicométrico do Teste Informatizado de Percepção de Emoções Primárias. Avaliação Psicológica, 13(1), 1-9. Recuperado em http:// pepsic.bvsalud.org/scielo.php?pid=S1677$-04712014000100002 \&$ script $=$ sci_arttext

Mihura, J. L. (2012). The necessity of multiple test methods in conducting assessments: The role of the Rorschach and self-report. Psychological Injury and Law, 5(2), 97-106. doi:10.1007/ s12207-012-9132-9

Nicolò, G., Semerari, A., Lysaker, P. H., Dimaggio, G., Conti, L., D’Angerio, S., ...Carcione, A. (2011). Alexithymia in personality disorders: Correlations with symptoms and interpersonal functioning. Psychiatry Research, 190(1), 3742. doi:10.1016/j.psychres.2010.07.046

Oliveira, J. H. A., \& Yoshida, E. M. P. (2009). Avaliação psicológica de obesos grau III antes e depois de cirurgia bariátrica. Psicologia: Reflexão e Crítica, 22(1), 12-19. doi:10.1590/S010279722009000100003

Paulhus, D. L., Lysy, D. C., \& Yik, M. S. M. (1998). Self-report measures of intelligence: Are they useful as proxy IQ tests? Journal of Personality, 66(4), 525-554. doi:10.1111/1467-6494.00023

Porcelli, P., Affatati, V., Bellomo, A., De Carne, M., Todarello, O., \& Taylor, G. J. (2004). Alexithymia and psychopathology in patients with psychiatric and functional gastrointestinal disorders. Psychotherapy and Psychosomatics, 73(2), 84-91. doi:10.1159/000075539
Sifneos, P. E. (1991). Affect, emotional conflict, and deficit: An overview. Psychotherapy and Psychosomatics, 56, 116-122. doi:10.1159/000288543

Torres, S., Guerra, M. P., Lencastre, L., Vieira, F., Roma-Torres, A., \& Brandão, I. (2011). Prevalência da alexitimia na anorexia nervosa e sua associação com variáveis clínicas e sociodemográficas. Jornal Brasileiro de Psiquiatria, 60(3), 182-189. doi:10.1590/S004720852011000300006

Veríssimo, R. (2011). Alexitimia: Da regulação afectiva na saúde e na doença. Lisboa, Portugal: Sítio do Livro.

Wagner, H., \& Lee, V. (2008). Alexithymia and individual differences in emotional expression. Journal of Research in Personality, 42(1), 8395. doi:10.1016/j.jrp.2007.04.001

Woyciekoski, C., \& Hutz, C. S. (2010). Inteligência emocional avaliada por autorrelato difere do construto personalidade? Psico-USF, 15(2), 151159. doi:10.1590/S1413-82712010000200003

Yoshida, E. M. P. (2000). Toronto Alexithymia Scale - TAS: Precisão e validade da versão em português. Psicologia: Teoria e Prática, 2(1), 59-74. Recuperado em http://editorarevistas.mackenzie.br/index.php/ptp/article/view/1119

Yoshida, E. M. P. (2007). Validade da versão em português da Toronto Alexithymia Scale-TAS em amostra clínica. Psicologia: Reflexão $e$ Crítica, 20(3), 389-396. doi:10.1590/S010279722007000300006
Recebido: 12/05/2014

$1^{a}$ revisão: $29 / 09 / 2014$

$2^{a}$ revisão: 05/10/2014 Aceite final: 08/10/2014 